\title{
A proactive system reliability analysis framework of electric vehicles
}

\author{
Xiaopin Zhong ${ }^{1}$, Wenwen Zeng ${ }^{2}$, Li Qiu ${ }^{1 *}$ \\ ${ }^{1}$ College of mechatronics and control engineering, Shenzhen university, \\ ${ }^{2}$ Shenzhen Key Laboratory of Micro-Nano Photonic Information Technology, Shenzhen university, \\ Shenzhen, China, \\ \{xzhong, zengww, qiuli\}@szu.edu.cn
}

\begin{abstract}
System reliability analysis (SRA) is a core support technique of many applications in the entire product lifecycle of electric vehicle (EV). However, the large-scale industrialization needs an accurate SRA due to the features of complexity, coupling and unknown noise of an EV. Besides component failure, the latent failure of electrical motor, electrical control and other subsystems is more originated from the uncertain dependency between components and the uncertain logical structures of failure. In this article, a proactive framework is presented to perform SRA on EVs. This framework is theoretically based on the probabilistic graphical model. Failure logical structures can then be recognized by a structure learning process of graphical model. Other SRA tasks and fault diagnosis are converted to the problems of probabilistic inference with uncertainty. Simulation examples demonstrate the effectiveness of our proposed framework.
\end{abstract}

Keywords-proactive system reliability analysis, graphical model, electronic control system, electric vehicle

\section{INTRODUCTION}

The development of Electric Vehicle (EV) is an important way to reduce pollution. Developed countries have started the mass production. For China, it is wise to improve the product reliability before EV's mass production. Although a lot of methods for system reliability analysis (SRA) have been developed, traditional SRA strategies are not suitable for EVs for at least three unsolved problems.

First, parametric distribution models are used in traditional representations. The assumption that the reliability data obeys a certain probabilistic distribution is often violated so that the robustness cannot be guaranteed. Second, traditional methods use deterministic logical structures of failure without considering "noisy gates" $[1,2,13]$. The logic gates are indeed noisy in EVs when exist unknown failure modes, uncertain parameters and unknown environmental influence. For this reason, the output densities from the failure logical structures are also uncertain. Third, traditional approaches remain to use fixed logical structures predefined by experts. It ignores the failure dependency between components in the same level. This is not realistic in EVs, e.g. the failure structure changes when the driving mode is changed.

It is then crucial for SRA in EVs to proactively construct a reasonable failure logical structure and to represent this structure in a flexible way. Fortunately, researchers have found a versatile methodology to deal with the dependency description and the uncertain noisy gates over the last decade, i.e. probabilistic graphical model (PGM). The PGM have many advantages over the classical SRA formalisms due to the capacity of dependency description and of data incorporation. In addition, we can easily propagate the uncertainties in a PGM unlike in conventional logic models, e.g. fault trees and event trees. In this research, therefore, we use the PGM as the theoretical framework to unify all of the SRA tasks into the problems of PGM learning and inference.

The published PGM studies applied to SRA are also known as Bayesian networks (BNs) [1,3]. They can be classified into mainly two categories according to the variable type. First, working state based BNs indicate the fail or work events defined in discrete space $[4,5,6,11]$. They estimate the distribution of system state deterministically by using conditional probability tables (CPTs) that is predefined and fixed. Second, time-to-failure (TTF) model based BNs assign a probabilistic description to TTF data of an item $[7,8,9,12]$. Although no CPT is needed, they do not consider the uncertainty of the model parameters. In our case, the PGM is a generalization of BNs. It is expected to capture the dependency between nodes in the same level.

The paper is organized as follows. The proposed framework is introduced in the next section followed by simulation examples. We draw conclusion in the last section.

\section{PROACTIVE RELIABILITY ANALYSIS FRAMEWORK}

The proposed framework is illustrated in Figure. 2. A graphical model is employed to describe the failure logical structure of reliability data acquired. The reliability data, time-to-failure in our simulation case, is modeled by parametric statistical models and the failure structure can be actively learned through structure learning algorithm. In this framework, fault diagnosis and other SRA tasks are converted to the inference process of PGM. In this section, the four main phases involved in the framework are explained respectively.

A. Data acquisition

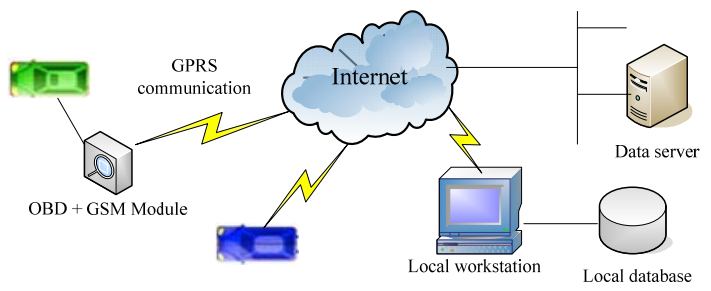

Figure 1. Data acquisition system 


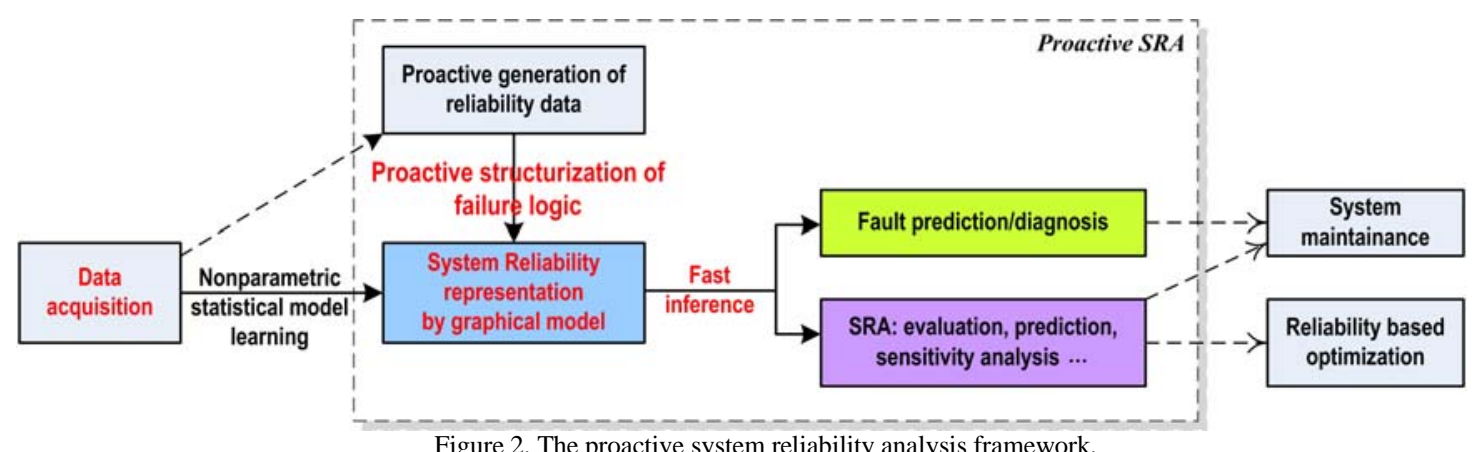

Figure 2. The proactive system reliability analysis framework.

Inside an electric vehicle, there are plenty electronic control subsystems monitored by the electronic control unit (ECU) and connected by a CAN bus. This feature allows to easily obtain reliability data from the on-board diagnostic (OBD) system. In order to adapt the moving property, we extended a GSM module to the OBD system so that the data can be sent back in real time by GPRS commu-nication through internet, see in Figure 1 for the data acquisition framework.

\section{B. System reliability representation by graphical model}

A directed acyclic network is built from the corresponding fault tree (FT) by Bobbi's method [1], see in Figure 3 for a simplest case.
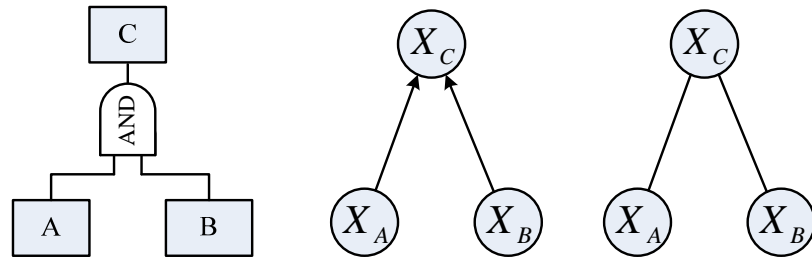

Figure 3. A simplest example for converting a FT (a) to a $\mathrm{BN}$ (b) and graphical model (c)

In our model, the network is undirected and the nodes denote the items' failure parameters that are the function outputs of the noisy gates rather than the deterministic gates. We denote a note's variable by $X_{i}, i \in I_{n}$, where $I_{n}$ is the label set of the notes. The time-to-failure (TTF) density is further denoted by $f_{i}\left(X_{i}\right)$, whose corresponding deterministic density is $f_{i}^{d}\left(X_{i}\right)$. Notice that a higher probability should be assigned to an uncertain density that is closer to $f_{i}^{d}$. We thus assume the distance between the two densities obeys a zero mean normal distribution, i.e.

$$
d\left(f_{i}\left(X_{i}\right), f_{i}^{d}\right) \sim N\left(0, \sigma_{i}\right), i \in I_{n},
$$

where $\sigma_{i}$ is the standard deviation encoding the dependency between the parameters of the basic items and those of the higher level item. A number of techniques can be used to estimate this deviation, such as maximum likelihood estimation (MLE).
Note that a distance cannot be negative. We further consider a one-sided normal distribution, i.e.

$$
\operatorname{Pr}\left[d\left(f_{i}\left(X_{i}\right), f_{i}^{d}\right) \mid \sigma_{i}\right]=\frac{2}{\sqrt{2 \pi} \sigma_{i}} \exp \left\{-\frac{d^{2}\left(f_{i}\left(X_{i}\right), f_{i}^{d}\right)}{2 \sigma_{i}^{2}}\right\} .
$$

There are choices for the distance between densities after discretizing, such as Euclidean distance and city-block distance [17].

For the deterministic density, it is in fact the output derived from the structure function. For a series structure, the system works only when all components function, i.e.

$$
f_{i}^{d}=\sum_{j \in B(i)} f_{j}\left(x_{j}\right) \prod_{k \in B(i) \backslash j}\left(1-F_{k}\left(x_{k}\right)\right), \quad i \in I_{n},
$$

where $B(i)$ denotes the label set of node $i$ 's basic component nodes and $F_{k}\left(X_{k}\right)$ is the cumulative probability of $X_{k}$. For a parallel structure, the system works if any component functions, i.e.

$$
f_{i}^{d}=\sum_{j \in B(i)} f_{j}\left(x_{j}\right) \prod_{k \in B(i) \backslash j} F_{k}\left(x_{k}\right), \quad i \in I_{n} .
$$

So far, we build a probabilistic graphical model with a detailed causal interpretation between the output parameters and those of basic items.

\section{Proactive structurization of failure logic}

It is not suitable for EVs to use the predefined failure structures for at least two reasons. First, the failure structure may change in EV-like complex systems. Second, it takes much time to define a failure structure and even experienced experts cannot guarantee the structure is correct. Therefore we turn to build the structure from the captured failure samples. It can be accomplished by data mining algorithms, such as K2 [10]. This allows the framework to adapt to the running mode of EVs.

\section{Fast inference}

After modeling, the network with continuous variables needs an approximate probabilistic reasoning process to calculate the posterior marginal probability of each item's parameters. To reduce the computation burden is still a big topic, although many explored techniques give good approxi -mation, such as dynamic discretization and mixtures of truncated exponentials, the readers are referred to $[14,15,16]$. Compared with other methods, Markov chain Monte Carlo 
(MCMC) is so far one of the mostly used techniques. It is suitable for the non-parametric densities employed in our model and some toolboxes can be easily applied. To make it converge faster, one of our future works is to consider an artificial-intelligence based MCMC, so called evolved MCMC.

\section{SIMULATION EXAMPLES}

In this section, we demonstrate simulations on the simplest model shown in Figure 3. Without loss of generality, we assume that $X_{A}, X_{B}$ and $X_{C}$ denote the failure rate of three components. Their actual values are given as $0.105,0.68$ and 0.785 respectively. Hundreds of i.i.d. observations can then be sampled from the corresponding exponential distribution of TTF.

If the data is modeled only by one-parameter (failure rate) exponential distribution, we evaluate respectively the normalized likelihoods and the marginal posteriors of each node. The results as well as $90 \%$ confidence interval of reliability are shown in Figure 4. If the basic nodes (A and B) are modeled by exponential distributions and that of the node $\mathrm{C}$ is modeled by a two parameter Weibull distribution.

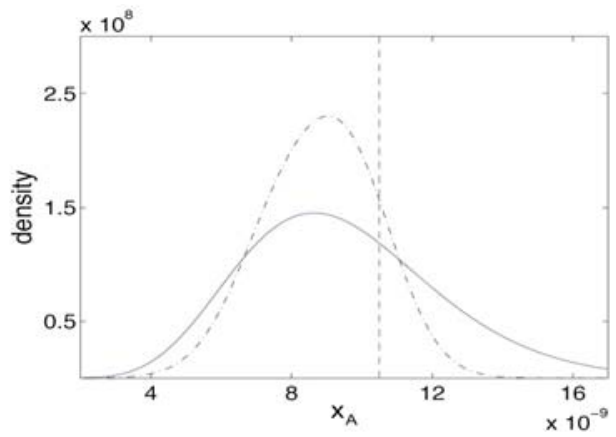

(a)

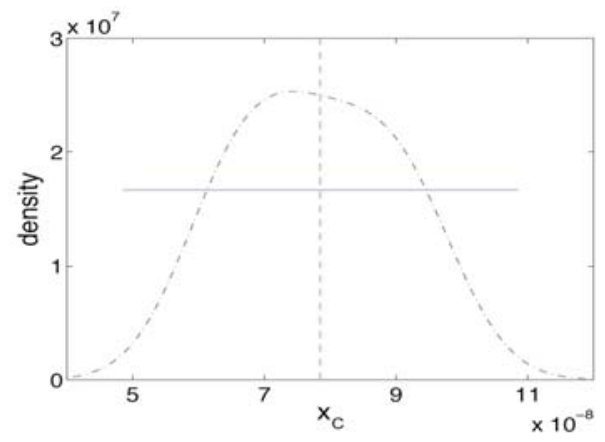

(c)
The results of the likelihood and the marginal posterior are shown in Figure 5.

We can observe the marginal probabilities have estimates (estimated by minimum mean square estimation or maximum a posterior) closer to the actual values than the likelihoods do. It demonstrates that the inference process gives a "better" guess of model parameters. This confirms the effectiveness of our framework.

\section{CONCLUSIONS}

This is a preliminary attempt to unify the tasks of system reliability analysis into a probabilistic graphical model. In the framework, we model the time-to-failure of components by the parametric distributions with random parameters. Their uncertainties learned from samples can be propagated within the network so that the reliability estimation, sensitivity analysis, fault diagnosis are all converted to the inference process of graphical model. This is demonstrated in the simulation examples. The proposed framework also allows us to construct the system's failure structure without intervention of experts who are expensive and make mistakes from time to time. The future work is to consider nonparametric statistical models in the framework.

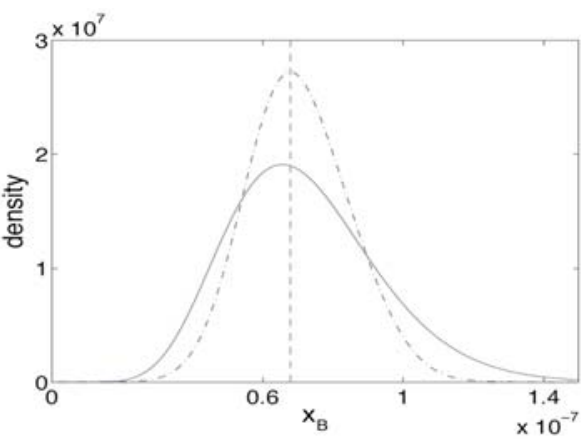

(b)

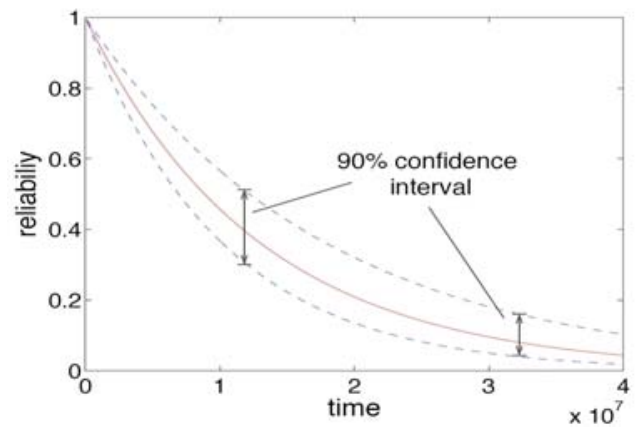

(d)

Figure 4. Normalized likelihood and marginal posterior of the three nodes' failure rates. The solid lines stands for the likelihoods; the dash-dot lines represent the posteriors and the dashed lines for the actual values. (d) shows the $90 \%$ confidence interval of reliability by the posterior mean. 


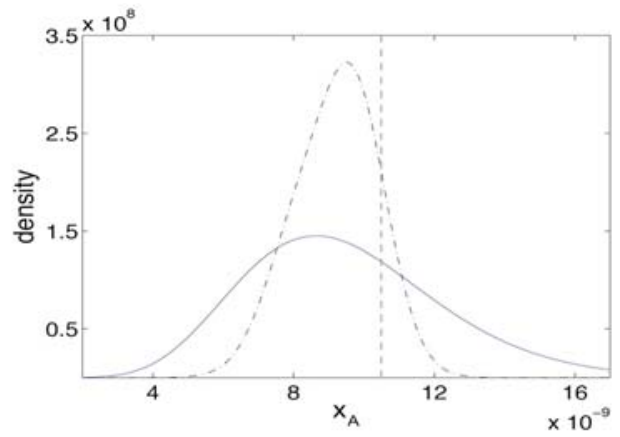

(a)

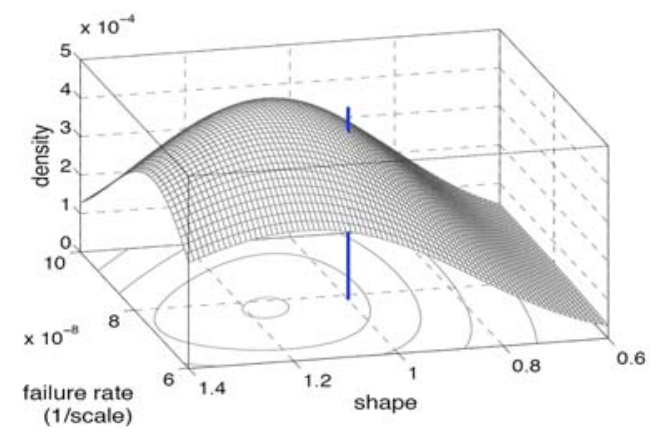

(c)

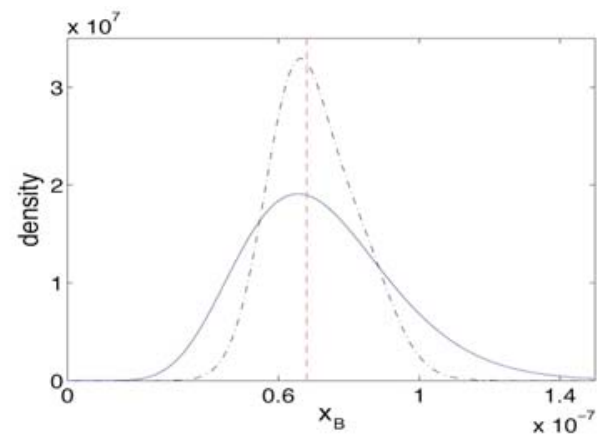

(b)

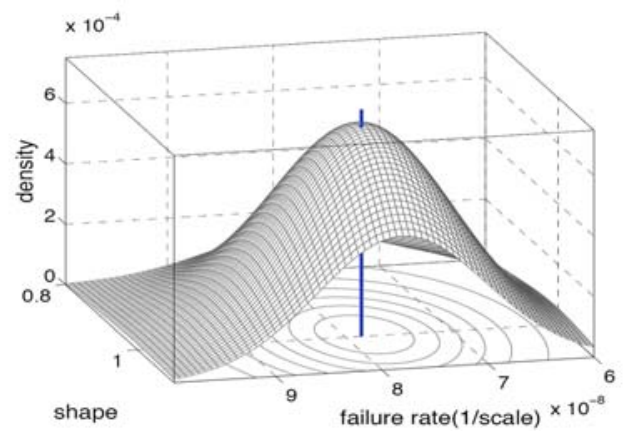

(d)

Figure 5. Likelihood and marginal posterior of $X_{2}$ with respect to Weibull parameters (shape and 1/scale). The blue lines indicate the actual values.

\section{ACKNOWLEDGEMENTS}

This research was supported by the National Natural Science Foundation of China under contract number 61203184, the Guangdong Natural Science Foundation under contract number S2012 040007116, and the Science, Technology and Innovation Commission of Shenzhen (Project No.JCYJ20120614085410435). The authors would also like to thank the anonymous reviewers.

\section{REFERENCES}

[1] Bobbio A, Portinale L, Minichino M, and Ciancamerla E. Improving the analysis of dependable systems by mapping fault trees into Bayesian networks. Reliability Engineering and System Safety, vol.71(3):249-260, 2001

[2] Wallstrom TC. Quantification of margins and uncertainties: A probabilistic framework, Reliability Engineering \& System Safety, Volume 96, Issue 9, September 2011, Pages 1053-1062

[3] Langseth H, Portinale L. Bayesian networks in reliability. Reliability Engineering and System Safety, vol.92(1):92-108, 2007

[4] Mahadevan S, Zhang R, Smith N. Bayesian networks for system reliability reassessment. Structural Safety, vol.23(3):231-251, 2001

[5] Torres-Toledano JG, Sucar LE. Bayesian networks for reliability analysis of complex systems. Lecture notes in artificial intelligence, vol.1484:195-206, 1998

[6] Wilson AG, Huzurbazar AV. Bayesian networks for multilevel system reliability. Reliability Engineering \& System Safety, vol.92(10):1413- 1420, 2007

[7] Boudali H, Dugan JB. A continuous-time Bayesian network reliability modeling, and analysis framework. IEEE Transactions on Reliability, vol.55(1):86-97, 2006

[8] Johnson VE, Graves TL, Hamada M, Reese CS. A hierarchical model for estimating the reliability of complex systems. In proceedings of the seventh Bayesian statistics international meeting, 199-213, Valencia, 2003

[9] Anderson-Cook CM, Graves T, Hamada M, Hengartner N, Johnson V, Reese CS, Wilson AG. Bayesian stockpile reliability methodology for complex systems with application to a missile stockpile. Technical report, Los Alamos National Laboratory LA-UR-05-1742, 2005

[10] Doguc O, Ramirez-Marquez JE. A generic method for estimating system reliability using Bayesian networks. Reliability Engineering and System Safety 2009;94(2):542-50

[11] Weber P, Jouffe L. Complex system reliability modelling with dynamic object oriented Bayesian networks (DOOBN). Reliability Engineering and System Safety 2006;91(2):149-62.

[12] Neil M, Tailor M, Marquez D, Fenton N, Hearty P. Modelling dependable systems using hybrid Bayesian networks. Reliability Engineering and System Safety 2008;93(7):933-9.

[13] Zafiropoulos EP, Dialynas EN. Reliability and cost optimization of electronic devices considering the component failure rate uncertainty. Reliability Engineering and System Safety 2004;84(3):271-84.

[14] Langseth H, Nielsen TD, Rumı'R, Salmero'n A. Inference in hybrid Bayesian networks. Reliability Engineering and System Safety 2009;94(10):1499-509.

[15] Moral S, Rumf R, Salmero 'n A. Mixtures of truncated exponentials in hybrid Bayesian networks. In: Proceedings of symbolic and quantitative approaches to reasoning with uncertainty: 6th European conference, ECSQARU 2001. Springer Verlag; 2001. p. 156-67.

[16] Marquez D, Neil M, Fenton N. Improved reliability modeling using Bayesian networks and dynamic discretization. Reliability Engineering and System Safety 2010;95(4):412-25.

[17] Cha SH. Comprehensive survey on distance/similarity measures between probability density functions. International Journal of Mathematical Models and Methods in Applied Sciences 2007;1(4):300-7. 